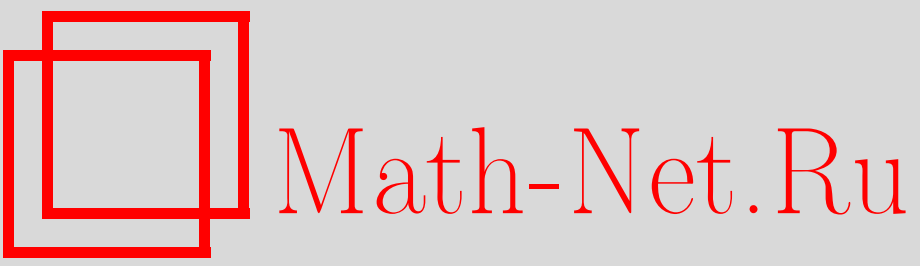

Г. С. Чакветадзе, Эргодические свойства инвариантных мер косого произведения в одномерной модели бурения, УМH, 1997, том 52, выпуск 4, 223-224

DOI: https://doi.org/10.4213/rm880

Использование Общероссийского математического портала Math-Net.Ru подразумевает, что вы прочитали и согласны с пользовательским соглашением

http://www.mathnet.ru/rus/agreement

Параметры загрузки:

IP: 54.209 .52 .79

26 апреля 2023 г., 18:31:35 


\title{
ЭРГОДИЧЕСКИЕ СВОЙСТВА ИНВАРИАНТНЫХ МЕР КОСОГО ПРОИЗВЕДЕНИЯ В ОДНОМЕРНОЙ МОДЕЛИ БУРЕНИЯ
}

\author{
Г. ЧАКВЕТАДЗЕ
}

Исследуется модель бурения, предложенная А. Лясотой в [1]. Чтобы определить одномерное преобразование, описьвающее динамику процесса бурения, введем отображения $v: \mathbb{R} \mapsto \mathbb{R}$ и $w:[0,1) \mapsto[0,1)$. Пусть $p: \mathbb{R} \mapsto \mathbb{R}-1$-периодическая, выпуклая и дважды непрерывно дифоференцируемая на $[0,1]$ функция, симметричная относительно точки $\frac{1}{2}, m=\inf _{r \in[0,1]}\left|p^{\prime \prime}(r)\right|$, $M=\sup _{r \in[0,1]}\left|p^{\prime \prime}(r)\right|, \lambda<m$. Для $s \in \mathbb{R} v(s)=v_{\lambda}(s)$ - абсцисса ближайшей справа к $s$ точки пересечения граффиков функций $p$ и $u_{s}$, где

$$
u_{s}(r)=u_{\lambda}(r, s)=p(s+0)+p^{\prime}(s+0)(r-s)-\frac{\lambda}{2}(r-s)^{2} .
$$

Отображение $w$ задается равенством

$$
w(s)=w_{\lambda}(s)=v(s) \bmod 1, \quad s \in[0,1) .
$$

Объектом изучения является преобразование $\tau:[0, N) \mapsto[0, N)$, определяемое по формуле

$$
\tau(s)=\tau_{\lambda, N}(s)=w(s \bmod 1)+[v(s)] \bmod N, \quad s \in[0, N), \quad N \in \mathbb{N}
$$

( $[r]$ обозначает целую часть числа $r$ ).

Опишем кратко геометрические свойства отображений $w$ и $\tau$ в предположении, что $\lambda<\frac{m}{2}$.

ЛЕмма 1. $\exists$ точки $s_{0}, s_{1}, \ldots, s_{n}, n \geqslant 2,0=s_{n}<s_{n-1}<\cdots<s_{1}<\frac{1}{2}<s_{0}=1$ maкue, что отображение $w$ непрерывно дифференцируемо на интервалах $I_{k}=\left(s_{k}, s_{k-1}\right), k=$ $1, \ldots, n$, и строго убьвает на $I_{1}$ от $1-s_{1}$ до 0 , на $I_{k}, k=2, \ldots, n-1,-$ от $1-s_{k}$ до $w \circ w\left(s_{k-1}+0\right)$, ина $I_{n}-$ от $w(0+0)$ до $w \circ w\left(s_{n-1}+0\right)$, причем $w\left(\frac{1}{2}\right)<\frac{1}{2}$.

Лемма 2. Для $s \in\left(s_{k}+i-1, s_{k-1}+i-1\right), k=1, \ldots, n, i=1, \ldots, N$, справедлива формула

$$
\tau(s)=w(s-i+1)+(k+i-1) \bmod N .
$$

Для $p \geqslant 1$, множества $E=\coprod_{j=1}^{t}\left[c_{j}, d_{j}\right]$ и функции $f$, заданной на $E$, символом $V_{E}^{p} f$ обозначается число $\sum_{j=1}^{t} V_{\left[c_{j}, d_{j}\right]}^{p} f$, где $V_{\left[c_{j}, d_{j}\right]}^{p}-p$-вариация $f$ на $\left[c_{j}, d_{j}\right] ; E$ назьвается $\tau$-инвариантным, если $\tau(E)=E \bmod 0$.

Tеорема 1. Пусть $V_{[0,1]}^{1} p^{\prime \prime}<\infty$ и выполнено условие $(m-\lambda)^{3} / \lambda>(M-\lambda)^{2}+$ $\frac{1}{8}(M-m)(m-\lambda)$. Тогда $\forall N$ отображсение $\tau$, определяемое по формуле $(\mathrm{A})$, имеет конечную абсолютно непрерывную инвариантную меру $\mu$, причем все такие меры сосредоточены на $\tau$-инвариантном множестве $G=\coprod_{i=0}^{N-1}\left[w\left(1-s_{n-1}\right)+i, 1-s_{n-1}+i\right]$.

Пусть $\mu_{w}$ - конечная абсолютно непрерывная инвариантная мера отображения $w$ (см. [2]). Преобразование $\tau$ изоморфно косому произведению с $N$-точечным слоем над $w$. Через $\mu_{N}$ обозначим равномерную меру на множестве $\{1, \ldots, N\}$. Изоморфизм переводит инвариантную меру $\mu_{w} \times \mu_{N}$ косого произведения в требуемую меру $\mu$. Вторая часть утверждения теоремы следует из того факта, что множество $F=\left[w\left(1-s_{n-1}\right), 1-s_{n-1}\right] w$-инвариантно и $\forall s \in(0,1)$ $\operatorname{dist}\left(w^{j}(s), F\right) \stackrel{j \rightarrow \infty}{\longrightarrow} 0$.

Исследуем эргодические свойства системы $(\tau, \mu)$. Пусть $\tau_{G}=\left.\tau\right|_{G}$. В пространстве ограниченных измеримых функций на $G$ рассмотрим оператор Перрона-Фробениуса $P_{\tau}$, задаваемый формулой

$$
P_{\tau} f(y)=\sum_{s \in \tau_{G}^{-1}(y)} \frac{f(s)}{\left|\tau_{G}^{\prime}(s)\right|} .
$$

Оператор $P_{\tau}$ продолжается единственным образом до ограниченного линейного положительного оператора на $L_{1} \stackrel{\text { def }}{=} L_{1}(G, \nu)$, где $\nu$ - мера Лебега. 
Tеорема 2. Eсли $V_{[0,1]}^{1} p^{\prime \prime}<\infty u(m-\lambda)^{3} / \lambda>(M-\lambda)^{2}$, mo $\forall N$ (1) мера $\mu$ из теоремы 1 единственна; (2) динамическая система $(\tau, \mu)$ слабо перемешивает.

Пусть $T$ - индуцированное кусочно-монотонное отображение преобразования $\tau$ на множестве

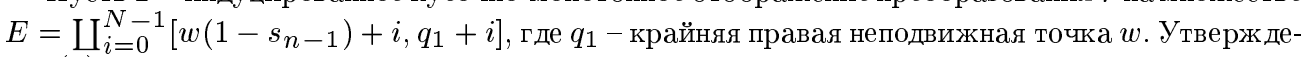
ние (1) выводится из следующего факта.

Лемма 3. Пусть

(i) $\inf _{E}\left|T^{\prime}\right|>1 u V_{E}^{1} \frac{1}{\left|T^{\prime}\right|}<\infty$;

(ii) $\inf \left|T^{\prime}\right|>2$ на $\coprod_{i=0}^{N-1}\left[w\left(1-s_{n-1}\right)+i, q_{2}+i\right]$, где $q_{2} \in I_{2}-$ прообраз $q_{1}$ при отображснии $w$;

(iii) $w\left(1-s_{1}\right)<q_{2}$.

Тогда $\forall N$ отображение $T$ эргодично.

В силу (i) в нашей ситуации применима теорема Вонга (см. [3]) о структуре разбиения на эргодические компоненты отображения $T$, в соответствии с которой число таких компонент конечно и каждая из них - конечное дизъюнктное объединение отрезков. Условия (ii) и (iii) позволяют уточнить эту структуру, используя понятие накрьвающей эргодической компоненты:

ОПРеДЕЛЕНИЕ. Эргодическая компонента $\Lambda$ отображения $T$ назьвается накрывающей, если $\exists i \in\{0, \ldots, N-1\}: q_{1}+i \in \Lambda$.

Оказьвается, что в условиях теоремы 2 накрывающие компоненты содержат в своей внутренности определенную область фазового пространства. Эргодичность преобразования $T$ следует из того факта, что при этом все эргодические компоненты $T$ - накрьвающие.

Теперь докажем (2). Сначала приведем следующее

Предложение 1. $\exists L \in \mathbb{N}: \inf _{G}\left|\left(\tau_{G}^{L}\right)^{\prime}\right|>1$. Кроме того, $V_{G}^{1} \frac{1}{\left|\left(\tau_{G}^{L}\right)^{\prime}\right|}<\infty$.

Положим $E_{l}=\left\{f \in L_{1}: P_{\tau}^{l} f=\lambda f, \lambda \in \mathbb{C},|\lambda|=1\right\}, l \in \mathbb{N}$. В силу предложения подпространства $E_{l}$ могут быть описаны с помощью теоремы Хофбауера и Келлера о спектральном разложении оператора $P_{\tau}$ (теорема 1 в [4]), и $\forall l \in \mathbb{N}$ выполнено $E_{l}=E_{1}, \operatorname{dim} E_{1}<\infty$. Так как точки спектра оператора $P_{\tau}$, принадлежащие единичной окружности, порождают в ней конечную подгруппу (см. [5]), то $\exists K \in \mathbb{N}: E_{K}=\left\{f: P_{\tau}^{K} f=f\right\}, \inf _{G}\left|\left(\tau_{G}^{K}\right)^{\prime}\right|>1$. С этого момента рассуждаем по схеме, предложенной в доказательстве леммы 3 , с $\tau_{G}^{K}$ вместо $T$ (роль оценок (ii) и (iіi) при этом играет условие эргодичности, полученное в (1)) и приходим к тому, что $\operatorname{dim} E_{1}=1$. Слабое перемешивание вытекает из теоремы 2 в [4].

Из теоремы 2 и теорем $3-5$ в [4] выводится, что эндоморфизм $(\tau, \mu)$ точный, бернулиевский и, если $V_{G}^{p} f<\infty, p \geqslant 1$, то для стохастического процесса $S_{u}=\sum_{j=0}^{u-1} f \circ \tau^{j}$ выполнена центральная предельная теорема.

Автор глубоко признателен проф. Степину за поддержку и интерес к этой работе.

\section{СПИСОК ЛИТЕРАТУРЫ}

[1] Lasota A., Rusek P. // Archivum Gornictwa. 1974. V. 19. № 3. P. 281-295. [2] Chakvetadze G. // J. Dynamical and Control Systems. 1996. V. 2. №4. P. 485-502. [3] Wong S. // Trans. Amer. Math. Soc. 1979. V. 246. P. 493-500. [4] Hofbauer F., Keller G. // Math. Z. 1982. V. 180. P. 119-140. [5] Rychlik M. // Studia Math. 1983. P. 69-80. 